\title{
Optical Coherence Tomography of Peri-Ocular Skin Cancers: An Optical Biopsy
}

\author{
Sabrina Bergeron ${ }^{a}$ Bryan Arthurs ${ }^{b}$ Debra-Meghan Sanft ${ }^{b}$ \\ Christina Mastromonaco ${ }^{a}$ Miguel N. Burnier Jr. ${ }^{a, b}$ \\ aThe MUHC, McGill University Ocular Pathology and Translational Research Laboratory, McGill University, \\ Montreal, QC, Canada; b Department of Ophthalmology, McGill University, Montreal, QC, Canada
}

\section{Keywords \\ Adnexal tumours - Basal cell carcinoma - Dermatology · Eyelids · Histopathology · Non-melanoma skin cancer . Optical coherence tomography - Ocular pathology · Oculoplastics}

\begin{abstract}
Introduction: Optical coherence tomography (OCT) imaging has been used as a diagnostic tool for retinal disease for several years, and OCT apparatuses are becoming increasingly powerful. However, OCT has yet to reach its full potential in ophthalmology clinics. Alike retinal layers, it has been shown that OCT is able to generate cross-sectional images of the skin and allows visualization of skin lesions in a histopathology-like manner. Objective: We aim to validate OCT as an imaging modality for peri-ocular skin cancer. Through a series of cases, we highlight findings for 3 common eyelid malignancies: basal cell carcinoma, squamous cell carcinoma and sebaceous carcinoma. We propose an OCT imagebased signature for basal cell carcinoma. Methods: This is a prospective study. Fifty-eight lesions suspicious of malignancy from 57 patients were subjected to OCT imaging prior to the surgical excision of the lesion. OCT images were analysed and scored according to previously identified OCT fea-
\end{abstract}

tures. Eight representative examples are presented, highlighting the OCT patterns for each malignancy side by side to its corresponding histopathological sections. Results: Out of the 58 lesions analysed, 53 were malignant. A loss of the dermal-epidermal junction is observed in all malignant lesions. A strong link is observed between the presence of subepithelial hyporeflective nests on OCT and the diagnosis of basal cell carcinoma (present in $83 \%$ of cases). Conversely, lesions of epithelial origin such as squamous cell carcinoma are most often represented on OCT by acanthosis. Two supplementary cases, one basal cell carcinoma and one sebaceous carcinoma, are provided to illustrate how OCT imaging is a valuable tool in cases where clinical observations may be unusual. Conclusions: We provide evidence supporting the use of OCT for the evaluation of peri-ocular cancers. OCT enables visualization of the skin layers in vivo, before biopsy. Our results show that certain OCT features can contribute to include or exclude a diagnosis of basal cell carcinoma. By integrating this non-invasive imaging methodology into the routine assessment of peri-ocular skin lesions, especially in health care centres where access to specialists is limited, OCT imaging can increase clinical precision, reduce delays in patient referral and enhance patient care.

(c) 2020 S. Karger AG, Basel karger@karger.com

(c) 2020 S. Karger AG, Basel

www.karger.com/oop

Karger"
Sabrina Bergeron

McGill University

1001, boulevard Décarie, E02.2389

Montréal QC H4A 3J1 (Canada)

sabrina.bergeron@mail.mcgill.ca 


\section{Introduction}

Optical coherence tomography (OCT) has played a pivotal role in clinical ophthalmology and it has shaped our current understanding of retinal diseases. The available technologies are increasingly powerful, yet not fully exploited. Retina specialists use OCT imaging to visualize retinal layers in such details and in an orientation that is comparable to light or electron microscopy [1-3]. Our aim is to expand the use of OCT imaging to characterize peri-ocular skin cancers.

Like retinal layers, the skin's architecture can be visualized by OCT imaging. On OCT, alternating bands of variable reflectivity and thickness permits segmentation of the skin in 4 distinctive layers: the stratum corneum, the epithelium, the dermal-epidermal junction and the dermis $[4,5]$. Previous dermatological studies assessing OCT imaging of skin cancers have shown improved diagnostic precision and accuracy when OCT imaging was added to clinical observations [6-9]; however, none of them has used an ophthalmological OCT, and very few studies have validated their findings with histopathology $[10,11]$.

The incidence of non-melanoma skin cancer (NMSC) is rising, and it has been estimated that more than 1 million cases of NMSC are diagnosed per year [12]. The head and neck area is a preferential site, with $20 \%$ of them being concentrated on the eyelid or the peri-ocular region [13]. The slow growth of NMSC and the delicate nature of the peri-ocular area make them notoriously challenging to diagnose for the unexperienced observer. It has been estimated that 1 in 5 lesions will be clinically misdiagnosed $[14,15]$.

The 3 main types of peri-ocular NMSC are basal cell carcinoma (BCC), squamous cell carcinoma (SCC) and sebaceous carcinoma. BCC is a slowly growing, often asymptomatic, lesion presenting as papules or nodules and accounts for $80 \%$ of NMSC. The treatment of choice for peri-ocular BCCs is surgical excision of the lesion [13]. With clear surgical margins, BCC portends an excellent prognosis. SCC is the second most common form of NMSC and, with complete excision of the lesion, the reported recurrence rate is below $20 \%$ [16].

Sebaceous carcinoma is a rare entity; however, it tends to be reported as frequently, if not more often, than SCC in regard to the peri-ocular location [17]. Sebaceous carcinoma is an aggressive cancer with a greater invasive potential than BCC or SCC and a reported metastasis rate of $30 \%$ [17]. It is sometimes referred to as the "great masquerader" because of its ability to disguise as other benign or inflammatory conditions.
Patients presenting with peri-ocular skin cancers often require the attention of a specialist in oculoplastics. The diagnosis is based on clinical observation and validated by histopathology. One potential pitfall of eyelid biopsies is their small size and the risk of a false-negative result, which is currently estimated to $12 \%$ in non-specialized centres [18]. OCT imaging allows to visualize peri-ocular lesions with an architectural similitude to histology, hence facilitating patient referral and providing additional clinical information before biopsy.

Our aim is to demonstrate, by a series of cases, that OCT imaging can provide valuable information in the assessment of peri-ocular skin lesions and to correlate these images with their corresponding histopathological sections. Our study supports the concept of using traditional OCT to obtain an "optical biopsy."

\section{Methods}

This is a prospective study. Institutional Review Board approval was obtained, and patients aged 18 and over with peri-ocular lesions suspicious of skin malignancy were enrolled in the study (REB: BCC/2017-2608). Informed consent was obtained from 57 patients, in compliance to the tenets of the Helsinki Declaration. Clinical photos and OCT imaging before biopsy was obtained for a total of 58 peri-ocular lesions.

Clinical photographs of the lesions were obtained using a Nikon 1 AW 1 digital camera. Non-contact spectral-domain OCT imaging of the lesion and its corresponding contralateral side was performed using an Optovue RTVue, XR100, Avanti Edition (Fremont, CA, USA) equipped with the anterior-segment imaging module (L-CAM). Line scans covering the extent of the lesion as estimated clinically were obtained.

Surgical excision was performed by the treating oculoplastic surgeon (B.A.) and sent for histopathological examination (M.B.) as per standard of care. Histopathological HE sections were retrieved and digitalized using the Zeiss Axio Scan.Z1 scanner with a $\times 40$ objective. OCT images were subsequently compared to histopathology sections. The OCT features for each diagnosis will be carefully reviewed in an attempt to identify disease-specific patterns.

OCT images were assessed based on previously identified OCT features [19]: pronounced hyperreflective superior band, thickening of the epidermis, loss of dermal-epidermal junction, hyporeflective nodule (with or without surrounding halo), hyperreflective nodule, bunch-of-grapes nodules, cystic spaces and ulceration. As further discussed, for simplicity of analysis, the "bunch-of-grapes" criterion is merged with the "hyporeflective nodule" feature.

\section{Results}

Using spectral-domain OCT equipped with an anterior-segment module (Optovue, Fremont, CA, USA), we assessed 58 lesions of the peri-ocular area (53 malignan- 
Table 1. OCT features for each type of lesion

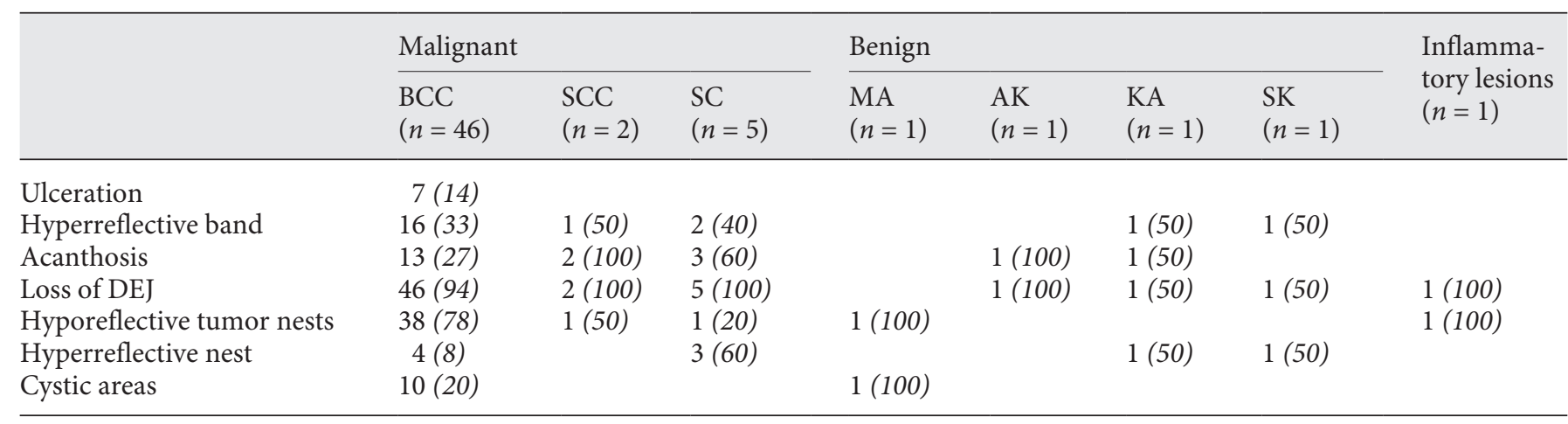

Table 2. Clinical, OCT and histopathological findings for each selected case

\begin{tabular}{|c|c|c|c|c|c|c|c|c|c|c|c|}
\hline \multirow[t]{2}{*}{ Case } & \multirow{2}{*}{$\begin{array}{l}\text { Age, } \\
\text { years }\end{array}$} & \multirow[t]{2}{*}{ Sex } & \multirow[t]{2}{*}{ Location } & \multirow{2}{*}{$\begin{array}{l}\text { Clinical } \\
\text { diagnosis }\end{array}$} & \multicolumn{6}{|l|}{ OCT feature } & \multirow[t]{2}{*}{ Histopathological diagnosis } \\
\hline & & & & & $\begin{array}{l}\text { hyperreflective } \\
\text { superior band } \\
\text { (hyperkeratosis) }\end{array}$ & $\begin{array}{l}\text { thickening of } \\
\text { epidermis } \\
\text { (acanthosis) }\end{array}$ & $\begin{array}{l}\text { loss of } \\
\text { DEJ }\end{array}$ & $\begin{array}{l}\text { hypo- } \\
\text { reflective } \\
\text { nodules }\end{array}$ & $\begin{array}{l}\text { hyper- } \\
\text { reflective } \\
\text { nodule }\end{array}$ & $\begin{array}{l}\text { cystic } \\
\text { spaces }\end{array}$ & \\
\hline 1 & 81 & M & RLL & $\mathrm{BCC}$ & & & Yes & Yes & & & BCC, nodular \\
\hline 2 & 69 & M & LLL & BCC & & Yes & Yes & Yes & & & BCC, nodular \\
\hline 3 & 87 & $\mathrm{~F}$ & LLL & $\mathrm{BCC}$ & & & Yes & Yes & & & BCC, nodular \\
\hline 4 & 71 & $\mathrm{~F}$ & RLL & $\mathrm{r} / \mathrm{o} \mathrm{SC}$ & Yes & & Yes & Yes & & & BCC, sclerosing \\
\hline 5 & 70 & $\mathrm{~F}$ & RLL & $\mathrm{BCC}$ & & & Yes & Yes & & & BCC, sclerosing \\
\hline 6 & 70 & M & RLL & SCC & Yes & Yes & Yes & & & & SCC, poorly differentiated \\
\hline 7 & 78 & $\mathrm{~F}$ & LLL & $\begin{array}{l}\text { SCC or } \\
\text { BCC }\end{array}$ & Yes & Yes & Yes & Yes & & & $\begin{array}{l}\text { SCC, malignant transformation } \\
\text { from keratoacanthoma }\end{array}$ \\
\hline 8 & 78 & M & RUL & $\begin{array}{l}\text { Mucinous } \\
\text { carcinoma }\end{array}$ & & & & Yes & & Yes & Mucinous carcinoma \\
\hline 9 & 81 & $\mathrm{~F}$ & RLL & BCC & & Yes & Yes & & Yes & & SC, invasive \\
\hline 10 & 78 & $\mathrm{~F}$ & LLL & SC & & & Yes & Yes & & Yes & BCC, sclerosing with cysts \\
\hline
\end{tabular}

DEJ, dermal-epidermal junction; RLL, right lower eyelid; LLL, left lower eyelid; RUL, right upper eyelid; BCC, basal cell carcinoma; SCC, squamous cell carcinoma; SC, sebaceous carcinoma.

cies, 4 benign and 1 inflammatory), including 46 BCCs, 5 sebaceous carcinomas and 2 SCCs. OCT features for each diagnosis are listed in Table 1.

Among the 53 malignancies, 10 representative cases were selected, each showing at least one, or multiple OCT features. Patient characteristics and OCT features are listed in Table 2. Figures 1-3 show an assemblage of clinical photos, OCT images and digitalized histopathological sections for each of the selected cases.

Cases 1-5 are BCCs, and all of them display a loss of the dermal-epidermal junction accompanied by hyporeflective nodules. The OCT image for case 2 (Fig. 1b) shows a BCC with thickening of the epidermis, corresponding to acanthosis on histopathology. Case 4 (Fig. 1d) 


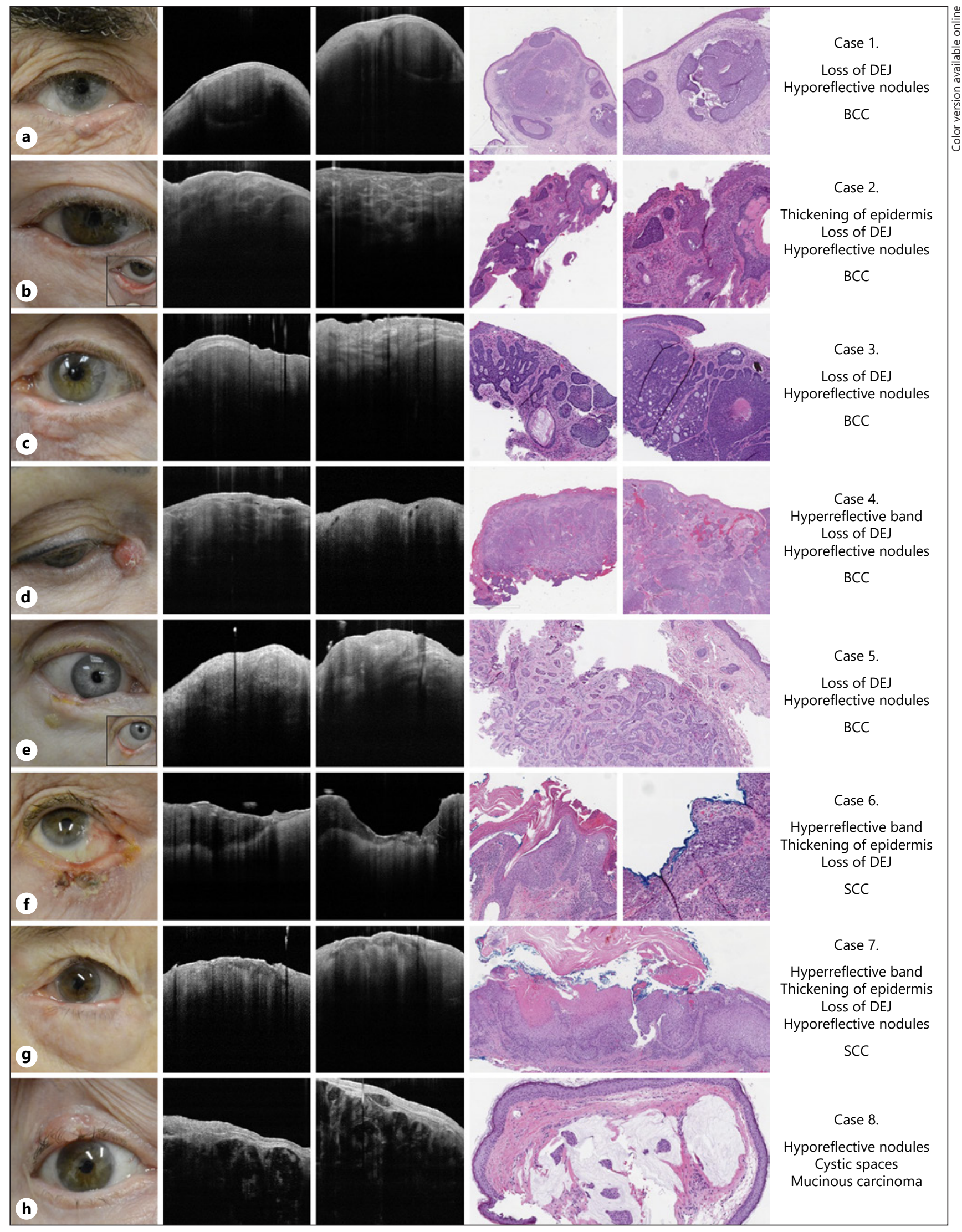

(For legend see next page.) 
shows mild hyperkeratosis, which is seen on OCT as a brighter and thicker than usual hyperreflective superior band. It is worth noting that we can also appreciate vascularization (clinically, telangiectasia) on the OCT image and histopathology. The possible presence of "halos" around certain hyporeflective nests is also an OCT observation of $\mathrm{BCC}$. These hyperreflective regions around the tumour nests are either the result of light scattering of the OCT signal, similarly to posterior shadowing on ultrasound or correspond to stromal degradation producing a myxoid stroma (further discussion of case 8 supports the latter).

Cases 6 and 7 (Fig. 1f, g), both SCCs, have OCTs showing a bright hyperreflective band, thickening of the epidermis and loss of the dermal-epidermal junction. This OCT pattern corresponds to hyperkeratosis, acanthosis and invasion of the epithelial neoplastic process, respectively. Of note, the OCT features for case 7 (Fig. 1g) could arguably suggest a BCC, because of the presence of hyporeflective nests; clinical impressions for this case were also BCC or SCC. In this particular lesion, the hyporeflective nests are above the dermal-epidermal junction, as opposed to underneath as in BCC. The histopathological diagnosis is an SCC arising from the malignant transformation of a keratoacanthoma.

Case 8 (Fig. 1h) shows hyporeflective nodules filled with a hyperreflective substance and cystic spaces. Histopathological correlation suggests that the prominent mucinous content of this lesion is responsible for the hyperreflective content within the hyporeflective nodules. This observation is supporting the previous hypothesis for the presence of bright halos surrounding the dark nodules in BCC, accounting for the heavy stromal degeneration or the myxoid stroma.

The next 2 examples are selected for their challenging clinical manifestations that may masquerade as other diseases, rendering clinical acumen difficult. Cases 9 and 10 highlight how integration of OCT imaging may help guide clinical decision prior to the surgical excision of the lesion.

Case 9 is a patient with a previous history of BCC and lentigo maligna, presenting with a right lower lid lesion

Fig. 2. Case 9, sebaceous carcinoma. a Clinical picture of the right lower lid lesion. b Overlay of dermoscopic image showing telangiectasia. c-d Representative OCT images. e-f HE-stained histopathological sections of the lesion showing large vacuolated neoplastic cells with prominent mitotic activity (not shown: adipophilin positivity and Ber-EP4 negativity).
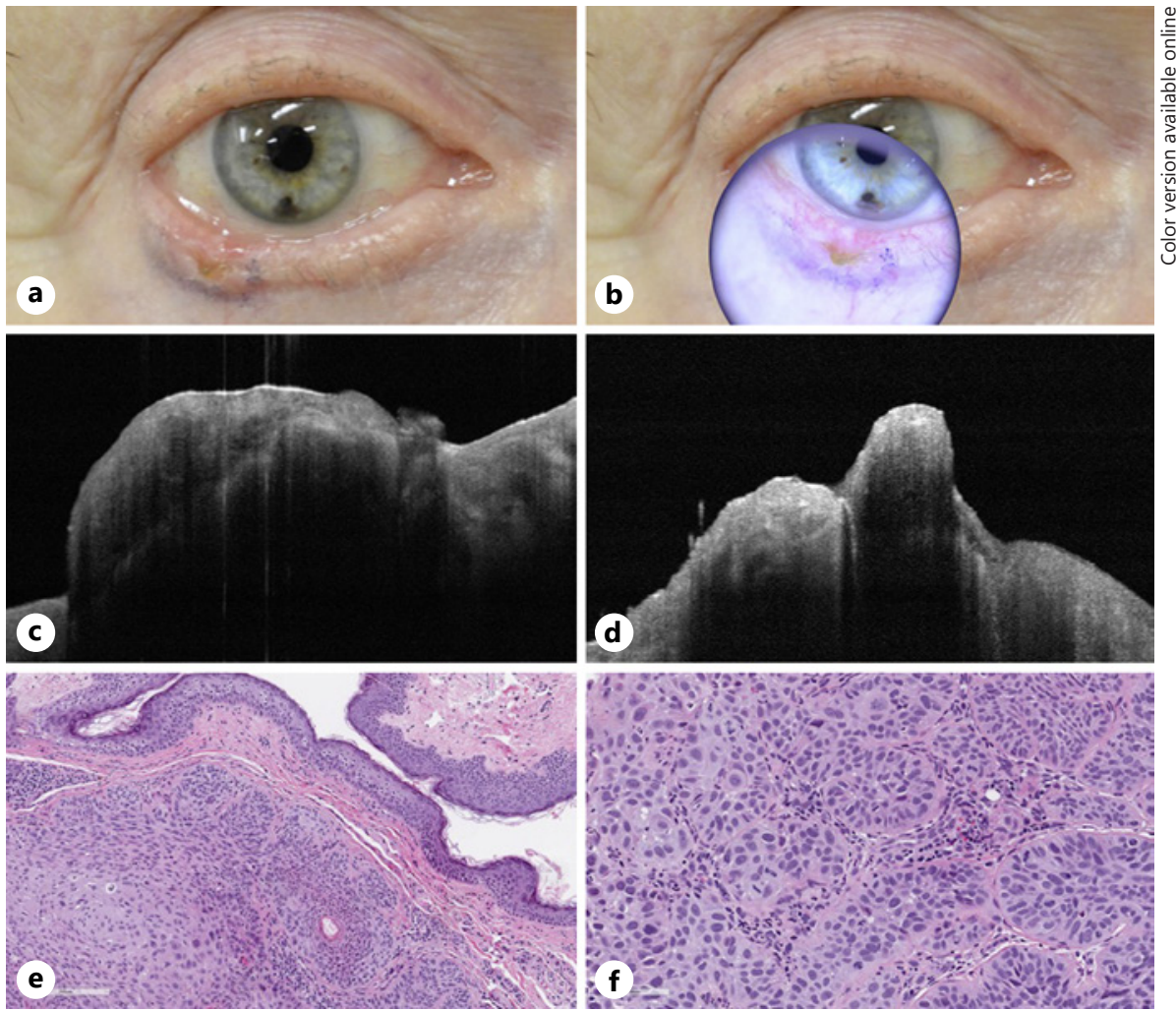

Fig. 1. Clinical assessment/OCT/histopathology correlation for cases 1-8. OCT images represent a 4-mm longitudinal area acquired from the largest tumour dimension. DEJ, dermal-epidermal junction; BCC, basal cell carcinoma; SCC, squamous cell carcinoma. 
Fig. 3. Case 10, basal cell carcinoma. a, b Clinical picture of the left lower lid lesion. c Representative OCT image showing hyporeflective nodules (yellow dotted circles) surrounded by hyperreflective halos (red dotted line) and a cyst (blue star). d-e HE-stained histopathological sections of the lesion showing islands and strands of basaloid cells with peripheral palisading, lymphocytic inflammatory infiltrate and large dilated vessels.
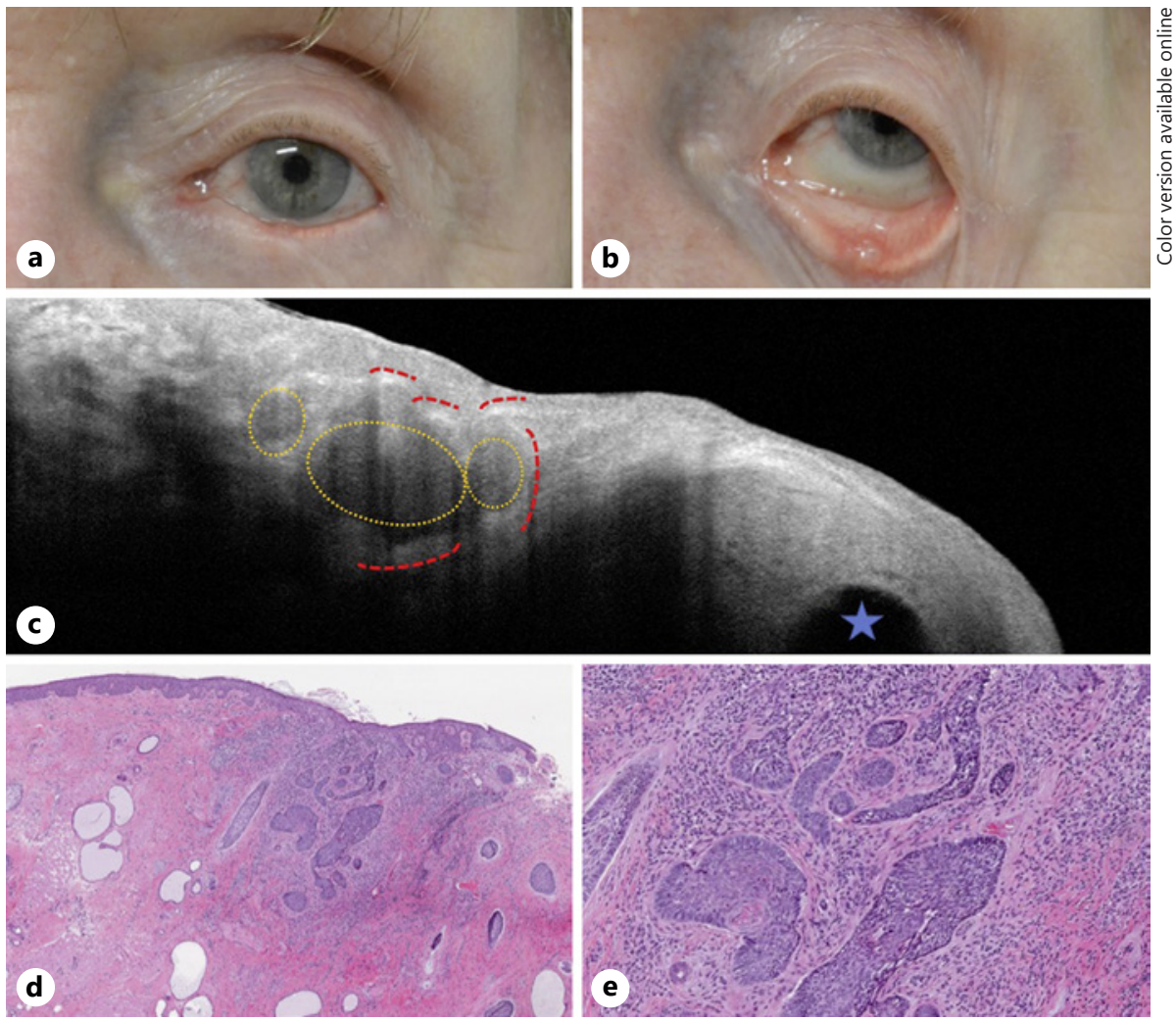

that shows ulceration and madarosis (Fig. 2a). A dermoscopic evaluation is performed and reveals telangiectasia as well as pearly elevated borders (Fig. 2b). Clinical manifestations are indicative of a BCC. Interestingly, OCT imaging of this lesion (Fig. 2c, d) does not show the typical BCC pattern (hyporeflective nodule) as presented in cases 1-5, suggesting a different diagnosis. Given the small size of the tumour, an excisional biopsy was performed. Histopathological analysis described the presence of large, vacuolated and pleiomorphic cells with numerous mitotic figures and positivity for the adipophilin immunohistochemical marker and negativity for BerEP4 (not shown), consistent with a sebaceous carcinoma of the lower eyelid (Fig. 2e, f).

Case 10 is a patient presenting with a left lower lid lesion that had been growing for 6 months. Lid eversion revealed a nodular mass with a yellowish background, suggestive of sebaceous carcinoma (Fig. 3a, b). Given the aggressiveness of sebaceous carcinoma, a full-thickness eyelid resection was performed and submitted for histopathological analysis. The pathology report was negative for sebaceous carcinoma and revealed a small focus of BCC surrounded by multiple cysts (Fig. 3d, e). Further examination of the OCT images is in support of this diagnosis; well-defined cystic spaces are present, as well as hyporeflective nodules surrounded with hyperreflective halos (Fig. 3c).

\section{Discussion}

Our results show that OCT imaging can provide valuable information in the initial assessment of peri-ocular cancers, both in first-line health care centres and in specialized eye care clinics. The advantages of adding OCT imaging to the routine assessment of peri-ocular skin cancers is 2-fold. (1) OCT imaging can increase diagnostic accuracy and favour patients' referral to oculoplastic services, and (2) OCT imaging can help to reduce the number of inconclusive biopsies.

Similarly to other groups who have successfully validated the use of traditional spectral-domain OCT to image ocular surface diseases [20-25], our aim is to provide an additional tool to assess peri-ocular skin lesions by using a technology that is already accessible in several eye care clinics. 
OCT images were analysed according to our previously described methodology, which is supported by other dermatological studies using OCT imaging $[19,26]$. Approximately 740 OCT images were acquired from the 58 lesions, as well as over 120 images from the normal contralateral skin. While the presence or absence of one single feature is not enough to make a conclusive diagnosis, we observe that each type points towards its own "OCT signature."

We identify an association between the presence of hyporeflective nodules and the diagnosis of BCC. Other criteria frequently observed in malignancies are "loss of dermal-epidermal junction," "acanthosis" and "hyperreflective nodule." However, these criteria are not exclusive to malignancies, and clinical correlation is essential. We also suggest the abolition of the previously described "bunch of grapes" feature, which was based on past literature and used to describe small nodules of low reflectivity [26]. Following our observation of all acquired OCT images, there is no specific histopathological finding associated with "bunch of grapes" other than they are small hyporeflective nodules. Therefore, we opt for merging it under the same umbrella category as hyporeflective nodules (applicable to nodules of all sizes).

We observe hyporeflective tumour nests in $83 \%$ of BCCs, while they are merely seen in other malignancies (Table 1), suggesting a strong association between hyporeflective nests and islands of basaloid cells on histopathology. The proposed signature for OCT of BCCs is the presence of hyporeflective nests underneath disruptions in the dermal-epidermal junction.

Both cases of SCC show a thick and bright superior band on OCT, which is histopathologically correlated to pronounced acanthosis. As it is demonstrated in Table 2, this finding is not pathognomonic of SCC as we can also observe acanthosis in several benign and malignant processes such as actinic keratosis, keratoacanthoma and sometimes in BCC and sebaceous carcinoma. An increased number of cases is required to better define OCT findings of SCC.

Similarly, our observations for sebaceous carcinoma are heterogeneous, sometimes displaying a thickened epithelium and/or hyperreflective nodules and halos. Given that sebaceous carcinoma arises from the tarsus in most cases, OCT imaging of this entity is currently limited to those involving the eyelid skin or the palpebral conjunctiva adjacent to the eyelid margin.

As more OCT images of peri-ocular skin lesions are obtained, refined OCT criteria and more "OCT signatures" for each differential diagnosis will be described.
This is especially useful for cases with atypical clinical presentations, such as the sebaceous carcinoma in case 9 and the BCC in case 10. The use of OCT imaging pointed towards the correct diagnosis in both cases, supporting the concept of using OCT to obtain an "optical biopsy" before proceeding to a surgical biopsy.

The main limitation is depth of imaging. By example, BCCs with pronounced hyperkeratosis may not show typical hyporeflective nests on OCT; this phenomenon is observed for 7 out of the 8 cases of BCC reported to not show hyporeflective nests. In these instances, OCT imaging reveals a thick hyperreflective superior band and posterior shadowing underneath. The OCT apparatus employed uses an 840-nm wavelength light source and limits the depth of skin imaging to $0.5-1 \mathrm{~mm}$. Other OCTs designed specifically for anterior-segment imaging are using a longer wavelength, which may offer a better penetration. However, the resolution of the image is lesser and ensues a loss of sensitivity.

Other teams composed mainly of dermatologists have attempted similar correlations using a different OCT technology, but their findings are restricted to only BCC or actinic keratosis $[27,28]$. More so, the OCT used in the dermatological setting is different than the one currently found in ophthalmology clinics as it requires contact with the skin. This is an undesirable quality for lesions that are in close proximity to the eye or at the eyelid margin. The observations from this prospective study are novel and we encourage other groups to also start using OCT technology to assess periocular skin lesions. As OCT imaging is painless and requires mere minutes, imaging during follow-up appointments is also recommended in order to pick up potential recurrences before they are apparent at the surface.

The interpretation of OCT images is user dependent, and a learning curve is to be expected. In an attempt to curb the interobserver discrepancies, other groups have established Likert-type grading systems $[29,30]$, but further studies are required to achieve a desirable diagnostic accuracy and sensitivity. It is also hypothesized that measurement of signal intensity and skin layer thickness may be yielding significant diagnosis information [31].

Dermatologists and computer scientists have teamed up and created algorithms capable of segmenting skin layers on OCT, thus enabling automated BCC detection by integrating deep learning and artificial intelligence in OCT imaging [32]. The application of such algorithms to current findings will undoubtedly cause a paradigm shift in how peri-ocular lesions are diagnosed. 
With the data accumulated so far, general recommendations for the interpretation of OCT images is as follows: (1) identify the normal epithelium, the dermal-epidermal junction and the dermis surrounding a lesion and (2) gather evidence of all features and analyse them in relation to their anatomical location within the skin. A hyporeflective mass in the dermis is strongly suggestive of a BCC, whilst a hyperreflective one may suggest a lesion of sebaceous origin. Abnormalities restricted to the epithelium are suggestive of squamous diseases; however, their reflectivity seems to vary depending on the level of differentiation of the lesion. A clinical correlation is essential for a successful interpretation.

\section{Conclusion}

In summary, our results provide substantial evidence that OCT imaging is a suitable tool to assess peri-ocular skin cancers. We identified specific patterns, or OCT signatures, that may help rule in or rule out certain diagnoses. Additional images will be required before OCT can be used to make a definitive diagnosis. Current observations will help the clinician have a better idea of the probable histopathological findings; hence the concept of "optical biopsy."

With the increasing incidence of skin cancer, we witnessed an unmet need for tools that allow for precision of diagnosis as well as early detection of skin lesions in delicate locations such as the eyelid. The new application for OCT imaging presented in this paper is rapid and noninvasive. Most importantly, OCT technology is well established, and spectral-domain technology is becoming more accessible in all types of clinical settings.

In the era of minimally invasive procedures and personalized medicine, OCT imaging offers a real-time visualization of skin lesions. As more images are obtained, our interpretation of these images will be refined. Whether its use leads to improved patient management will remain to be studied.

\section{Acknowledgements}

The research group would like to acknowledge all members of the MUHC - McGill University Ocular Pathology and Translational Research Laboratory, the residents of the McGill Department of Ophthalmology, as well as the support staff at the McGill Academic Eye Centre for their support and insightful views during this project.

\section{Statement of Ethics}

The research complies with the guidelines for human studies, and the research was conducted ethically in accordance with the World Medical Association Declaration of Helsinki. This is a prospective study approved by the McGill University Health Centre. Institutional Review Board approval was obtained (REB: BCC/2017-2608). Written informed consent and permission to use research data and images for publications was obtained from each patient prior to the study.

\section{Conflict of Interest Statement}

The authors have no conflict of interest to declare.

\section{Funding Sources}

No funding was obtained for this study.

\section{Author Contributions}

B.A., the treating physician, performed surgical excision of the lesions and made a major contribution in the writing of this manuscript. D.-M.S. participated in the elaboration of the research protocol and approval of the study as well as provided insightful clinical views and support during data acquisition. C.M. was responsible for coordinating the study and served as a liaison between the ethics review board and the research team. M.N.B. Jr. is the principal investigator and also performed the histopathological examination of all specimens. S.B., under the supervision of M.N.B. Jr., performed histopathological analysis of all specimens. S.B. was responsible for conducting the study, data analysis and writing of this paper.

\section{References}

1 Cuenca N, Fernández-Sánchez L, Sauvé Y, Segura FJ, Martínez-Navarrete G, Tamarit JM, et al. Correlation between SD-OCT, immunocytochemistry and functional findings in an animal model of retinal degeneration. Front Neuroanat. 2014 Dec;8:151.

2 Pang CE, Messinger JD, Zanzottera EC, Freund KB, Curcio CA. The Onion Sign in
Neovascular Age-Related Macular Degeneration Represents Cholesterol Crystals. Ophthalmology. 2015 Nov;122(11):2316-26.

3 Moreira-Neto CA, Bergeron S, Coblentz J, Zoroquiain $\mathrm{P}$, Maloney $\mathrm{S}$, Mastromonaco $\mathrm{C}$, et al. Optimizing optical coherence tomography and histopathology correlation in retinal imaging. Can J Ophthalmol. 2019 Apr;54(2):280-7.
4 Gambichler T, Jaedicke V, Terras S. Optical coherence tomography in dermatology: technical and clinical aspects. Arch Dermatol Res. 2011 Sep;303(7):457-73.

5 Welzel J, Lankenau E, Birngruber R, Engelhardt R. Optical coherence tomography of the human skin. J Am Acad Dermatol. 1997 Dec; 37(6):958-63. 
6 Cheng HM, Lo S, Scolyer R, Meekings A, Carlos G, Guitera P. Accuracy of optical coherence tomography for the diagnosis of superficial basal cell carcinoma: a prospective, consecutive, cohort study of 168 cases. Br J Dermatol. 2016 Dec;175(6):1290-300.

7 Markowitz O, Schwartz M, Feldman E, Bienenfeld A, Bieber AK, Ellis J, et al. Evaluation of Optical Coherence Tomography as a Means of Identifying Earlier Stage Basal Cell Carcinomas while Reducing the Use of Diagnostic Biopsy. J Clin Aesthet Dermatol. 2015 Oct; $8(10): 14-20$.

8 Wang KX, Meekings A, Fluhr JW, McKenzie G, Lee DA, Fisher J, et al. Optical coherence tomography-based optimization of mohs micrographic surgery of Basal cell carcinoma: a pilot study. Dermatol Surg. 2013 Apr;39(4): 627-33.

9 Holmes J, von Braunmühl T, Berking C, Sattler E, Ulrich M, Reinhold U, et al. Optical coherence tomography of basal cell carcinoma: influence of location, subtype, observer variability and image quality on diagnostic performance. Br J Dermatol. 2018 May; 178(5): 1102-10.

10 Pelosini L, Smith HB, Schofield JB, Meeckings A, Dhital A, Khandwala M. In vivo optical coherence tomography (OCT) in periocular basal cell carcinoma: correlations between in vivo OCT images and postoperative histology. Br J Ophthalmol. 2013 Jul;97(7):890-4.

11 Mogensen M, Nürnberg BM, Thrane L, Jørgensen TM, Andersen PE, Jemec GB. How histological features of basal cell carcinomas influence image quality in optical coherence tomography. J Biophotonics. 2011 Aug;4(78):544-51.

12 Bray F, Ferlay J, Soerjomataram I, Siegel RL, Torre LA, Jemal A. Global cancer statistics 2018: GLOBOCAN estimates of incidence and mortality worldwide for 36 cancers in 185 countries. CA Cancer J Clin. 2018 Nov;68(6): 394-424.

13 Rosner M. CHAPTER 15 - Basal cell carcinoma. In: Singh AD, Damato BE, Pe'er J, Murphree AL, Perry J, editors. Clinical Ophthalmic Oncology. Edinburgh: W.B. Saunders; 2007. pp. 76-80.
14 Hillson TR, Harvey JT, Hurwitz JJ, Liu E, Oestreicher JH, Pashby RC. Sensitivity and specificity of the diagnosis of periocular lesions by oculoplastic surgeons. Can J Ophthalmol. 1998 Dec;33(7):377-83.

15 Baxter JM, Patel AN, Varma S. Facial basal cell carcinoma. BMJ. 2012 Aug;345:e5342.

16 Rosner M. CHAPTER 16 - Squamous cell carcinoma. In: Singh AD, Damato BE, Pe'er J, Murphree AL, Perry J, editors. Clinical Ophthalmic Oncology. Edinburgh: W.B. Saunders; 2007. pp. 81-4.

17 Rosner M. CHAPTER 17 - Sebaceous gland carcinoma. In: Singh AD, Damato BE, Pe'er J, Murphree AL, Perry J, editors. Clinical Ophthalmic Oncology. Edinburgh: W.B. Saunders; 2007. pp. 85-9.

18 Carneiro RC, de Macedo EM, de Lima PP, Bonatti R, Matayoshi S. Is 2-mm punch biopsy useful in the diagnosis of malignant eyelid tumors? Ophthal Plast Reconstr Surg. 2012 Jul-Aug;28(4):282-5.

19 Bergeron S, Miyamoto D, Sanft DM, Burnier JV, Mastromonaco C, Romano AA, et al. Novel application of anterior segment optical coherence tomography for periocular imaging. Can J Ophthalmol. 2019 Aug;54(4):4317.

20 Alzahrani YA, Kumar S, Abdul Aziz H, Plesec T, Singh AD. Primary Acquired Melanosis: Clinical, Histopathologic and Optical Coherence Tomographic Correlation. Ocul Oncol Pathol. 2016 Apr;2(3):123-7.

21 Medina CA, Plesec T, Singh AD. Optical coherence tomography imaging of ocular and periocular tumours. Br J Ophthalmol. 2014 Jul;98(Suppl 2):ii40-6.

22 Venkateswaran N, Galor A, Wang J, Karp CL. Optical coherence tomography for ocular surface and corneal diseases: a review. Eye Vis (Lond). 2018 Jun;5(1):13.

23 Nanji AA, Sayyad FE, Galor A, Dubovy S, Karp CL. High-Resolution Optical Coherence Tomography as an Adjunctive Tool in the Diagnosis of Corneal and Conjunctival Pathology. Ocul Surf. 2015 Jul;13(3):226-35.
24 Venkateswaran N, Mercado C, Tran AQ, Garcia A, Diaz PF, Dubovy SR, et al. The use of high resolution anterior segment optical coherence tomography for the characterization of conjunctival lymphoma, conjunctival amyloidosis and benign reactive lymphoid hyperplasia. Eye Vis (Lond). 2019 Jun;6(1): 17.

25 Tran AQ, Venkateswaran N, Galor A, Karp CL. Utility of high-resolution anterior segment optical coherence tomography in the diagnosis and management of sub-clinical ocular surface squamous neoplasia. Eye Vis (Lond). 2019 Aug;6(1):27.

26 Meekings A, Utz S, Ulrich M, Bienenfeld A, Nandanan N, Fisher J, et al. Differentiation of Basal Cell Carcinoma Subtypes in MultiBeam Swept Source Optical Coherence Tomography (MSS-OCT). J Drugs Dermatol. 2016 May;15(5):545-50.

27 Hussain AA, Themstrup L, Jemec GB. Optical coherence tomography in the diagnosis of basal cell carcinoma. Arch Dermatol Res. 2015 Jan;307(1):1-10.

28 Ferrante di Ruffano L, Dinnes J, Deeks JJ, Chuchu N, Bayliss SE, Davenport C, et al.; Cochrane Skin Cancer Diagnostic Test Accuracy Group. Optical coherence tomography for diagnosing skin cancer in adults. Cochrane $\mathrm{Da}$ tabase Syst Rev. 2018 Dec;12:CD013189.

29 Wahrlich C, Alawi SA, Batz S, Fluhr JW, Lademann J, Ulrich M. Assessment of a scoring system for Basal Cell Carcinoma with multi-beam optical coherence tomography. J Eur Acad Dermatol Venereol. 2015 Aug; 29(8):1562-9.

30 Ulrich $\mathrm{M}$, von Braunmuehl $\mathrm{T}$, Kurzen $\mathrm{H}$, Dirschka T, Kellner C, Sattler E, et al. The sensitivity and specificity of optical coherence tomography for the assisted diagnosis of nonpigmented basal cell carcinoma: an observational study. Br J Dermatol. 2015 Aug;173(2): 428-35.

31 Schuh S, Kaestle R, Sattler E, Welzel J. Comparison of different optical coherence tomography devices for diagnosis of non-melanoma skin cancer. Skin Res Technol. 2016 Nov; 22(4):395-405

32 Yow AP, Srivastava R, Cheng J, Li A, Liu J, Schmetterer L, et al. Techniques and Applications in Skin OCT Analysis. Adv Exp Med Biol. 2020;1213:149-63. 